山्ل FRANÇAISE

$\gg \mathrm{DE}$

댈 PEAAGOGIE

\section{Revue française de pédagogie}

Recherches en éducation

182 | 2013

Connaissances et politiques d'éducation : quelles interactions?

\title{
Carnus Marie-France \& Terrisse André (dir.). Didactique clinique de l'EPS. Le sujet enseignant en question
}

Paris : Éd. EP\&S, 2013, 160 p.

Gérard Vergnaud

\section{(2) OpenEdition}

\section{Journals}

Édition électronique

URL : http://journals.openedition.org/rfp/4043

DOI : $10.4000 /$ rfp.4043

ISSN : 2105-2913

Éditeur

ENS Éditions

Édition imprimée

Date de publication : 28 août 2013

Pagination : 146-147

ISSN : 0556-7807

Référence électronique

Gérard Vergnaud, «Carnus Marie-France \& Terrisse André (dir.). Didactique clinique de l'EPS. Le sujet enseignant en question », Revue française de pédagogie [En ligne], 182 | 2013, mis en ligne le 28 août 2013, consulté le 22 septembre 2020. URL : http://journals.openedition.org/rfp/4043 ; DOI : https:// doi.org/10.4000/rfp.4043 
« talent» ou à la « mobilité ». Ainsi, les décisions des conseils de classe concernant l'avenir des élèves sont mises en relation avec ces logiques qui renvoient à la «culture » des établissements. Cette notion renvoie ici à la culture professionnelle, qui se construit de façon localisée, et met l'accent sur ce qui fait l'unité des pratiques des acteurs scolaires par comparaison entre établissements. Mais dans la mesure où les pratiques sont plurielles au sein d'un même établissement, il paraît difficile d'y associer la notion de culture là où des arrangements locaux précisent les rôles, au sein d'une segmentation professionnelle que la diversité des pratiques vient confirmer.

La reconnaissance de culture d'établissement permet la construction du concept de champ, en tant qu'il relève d'une théorie de la différenciation sociale, convoqué pour analyser les jugements d'orientation des enseignants. On se situe là dans une perspective relationnelle et structurale qui vise à comprendre pourquoi les acteurs institutionnels s'expriment sous la forme d'une lutte de concurrence entre les différentes composantes du champ. Mais peut-on lire les relations entre les établissements sous l'angle exclusif de la concurrence? On peut se demander en effet si le domaine scolaire étudié est l'aboutissement d'un processus progressif d'autonomisation qui l'amènerait à se doter de principes de hiérarchisation et de structuration spécifiques et lui permettrait de tracer les frontières du champ. L'espace scolaire enquêté - dominé étant trop peu structuré pour que le concept de champ tel que Bourdieu l'a forgé (Bourdieu, 1989) puisse être pertinent, Géraldine André plaide pour un « usage renouvelé » de ce concept (p. 123).

Pour conclure, cet ouvrage propose une sociologie de l'orientation scolaire qui place au centre de l'analyse les rapports de domination liés à l'appartenance de classe, à partir de l'analyse des transmissions des héritages familiaux ouvriers et des logiques des acteurs de l'institution. Ce travail, qui analyse finement les sens attribués par les enfants d'ouvriers aux études selon leur configuration familiale, ainsi que les contraintes dans lesquelles sont pris les jugements professoraux à leur égard, pourrait être prolongé dans deux directions pour nourrir une sociologie de l'orientation scolaire renouvelée, comme nous y invite Géraldine André. La première serait de prendre en compte le destin social ultérieur des filles et des garçons, pour contribuer à la réflexion sur les processus de sous-sélection ou de sursélection des élèves de milieux populaires qui paraissent constituer un élément important des rapports à l'orientation. La seconde demanderait à considérer la spécificité du champ pédagogique, soit analyser les processus d'apprentissages et de transmission des savoirs, en suivant la proposition de Basil Bernstein, cité par Jean-Yves Rochex et Jacques Crinon (2011) : « Ce qui manque aux théories de la reproduction culturelle, c'est l'analyse interne de la structure du discours lui-même »(Bernstein, 2007, p. 26).

Séverine Chauvel

École normale supérieure, centre Maurice Halbwachs

\section{BIBLIOGRAPHIE}

BERNSTEIN B. (2007). Pédagogie, contrôle symbolique et identité. Laval : Presses universitaires de Laval.

BOURDIEU P. (1989). La noblesse d'État, grandes écoles et esprit de corps. Paris : Éd. de Minuit.

MAUGER G. (2006). "Les transformations des classes populaires en France depuis trente ans ". In J. Lojkine, P. Cours-Salies \& M. Vakaloulis (dir.), Nouvelles luttes de classes. Paris : PUF, p. 30-42.

ROCHEX J.-Y. \& CRINON J. (dir.) (2011). La construction des inégalités scolaires. Au cœur des pratiques et des dispositifs d'enseignement. Rennes : Presses universitaires de Rennes, p. 173-198.

WILLIS P. (1978). "L'école des ouvriers". Actes de la recherche en sciences sociales, $n^{\circ} 24, p .50-61$.

CARNUS Marie-France \& TERRISSE André (dir.). Didactique clinique de l'EPS. Le sujet enseignant en question. Paris : Éd. EP\&S, 2013, 160 p.

Voici un ouvrage novateur, qui ouvre un champ de recherches sur l'éducation jusqu'alors peu développé et peu connu. La didactique clinique est présentée dans cet ouvrage comme une problématique de recherche pleine et entière : elle est appliquée aux enseignants d'EPS et est conduite de manière systématique auprès d'une vingtaine d'entre eux, dont l'enseignement concerne une dizaine de sports différents. C'est un projet ambitieux.

L'équipe d'André Terrisse et de Marie-France Carnus, qui s'est forgée et développée à l'IUFM de Toulouse, a réuni autour d'elle des chercheurs motivés. Ils ont adopté le point de vue de la psychanalyse pour solliciter et interpréter la parole d'enseignants, qui ont investi leur métier avec toute leur subjectivité : projet, histoire personnelle, ambition pour autrui. C'est cette subjectivité qu'il s'agit d'interroger. Une douzaine de chapitres traitent de plusieurs thèmes de l'activité des enseignants d'EPS, mais, derrière les différences entre les objets étudiés, existe un cadre théorique commun, que l'on retrouve dans chaque chapitre.

Trois concepts fondamentaux, empruntés surtout à Freud et Lacan, structurent la problématique de l'équipe, ceux de « déjà-là », d' « épreuve » et d' " après-coup », et permettent de donner du sens à d'autres idées importantes comme celles de « sujet supposé savoir » et d' "impossible 
à supporter ». Ce ne sont pas les concepts ordinaires de la didactique, et la question se pose donc de la facilité/difficulté de la communication de l'équipe avec la communauté des chercheurs et celle des enseignants. Certains lecteurs seront surpris par une problématique à leurs yeux «étrange ». Et pourtant l'ouvrage est très intéressant : passée la surprise initiale provoquée chez le lecteur par les catégories utilisées, celui-ci s'intéressera aux propos tenus par les enseignants dont le témoignage est recueilli, et qui illustrent les attitudes et les représentations plus ou moins conscientes qui orientent la subjectivité des enseignants dans leur activité.

Le « déjà-là » désigne les filtres hérités de l'expérience passée, qui modifient et biaisent les objectifs de l'enseignant ainsi que son interprétation des observations qu'il peut faire dans sa classe. La comparaison entre un entretien avant le cours et un entretien après met assez aisément en évidence le décalage entre les objectifs institués et ceux de l'enseignant. En d'autres termes, l'enseignant n'est pas maître dans son institution.

Le terme « épreuve » désigne l'activité de l'enseignant en situation, avec ce qu'elle comporte d'ajustement à l'imprévu et d'écart éventuel avec l'attente de l'enseignant. On n'est pas éloigné cette fois de l'idée de contrat didactique ? L'aprèscoup permet de mettre cet écart en évidence.

Si l'enseignant est « supposé savoir », il ne peut qu'être surpris par les difficultés qu'il rencontre et par son embarras pour les surmonter, mais il lui est aussi « impossible de supporter » certaines faiblesses des élèves, par exemple leur faible engagement dans l'activité sportive que, justement, il privilégie lui-même en raison de son histoire personnelle.

Mettre ces différents phénomènes en évidence dans une variété d'activités sportives comme la gymnastique, la boxe française ou le volley-ball suppose évidemment que l'on tienne compte des spécificités de chaque activité, mais, dans tous les cas le « je » de l'enseignant est bel et bien un facteur important d'orientation de son activité et de son jugement. L'expertise professionnelle n'est pas l'expérience personnelle, les deux semblant plutôt s'infléchir l'une l'autre.

Plusieurs autres thèmes intéressent l'équipe de didactique clinique, comme l'évaluation ou encore l'introduction dans les programmes de contenus nouveaux, l'éducation à la santé par exemple.

Les préoccupations concernant la personne de l'enseignant commencent à être étudiées de plusieurs manières dans la recherche, en raison de la dualité des fonctions de l'activité de travail, à la fois une fonction productive (effets sur le monde extérieur) et une fonction constructive (effets sur la personne elle-même) ; aussi en raison du retour réflexif sur l'action, qui donne à l'expérience personnelle un véritatable statut conceptuel ; aussi en raison de la défense personnelle que doit assurer l'enseignant au cours de son interaction avec les élèves.

Les considérations méthodologiques occupent une bonne partie des contributions à l'ouvrage, notamment les considérations concernant le choix et la construction des cas individuels retenus pour l'analyse. La didactique clinique de l'EPS représente donc, à mon avis, un apport original à la didactique, et ajouterai-je, à la psychologie.

Gérard Vergnaud

Université Paris 8-Vincennes-Saint-Denis, laboratoire Paragraphe

DOUSSOT Sylvain. Didactique de l'histoire. Outils et pratiques de l'enquête historienne en classe. Rennes: Presses universitaires de Rennes, 2011, 316 p.

L'ouvrage de Sylvain Doussot, rédigé à partir de sa thèse soutenue en 2009, marque une importante avancée pour la didactique de l'histoire. Il fait notamment progresser le débat sur la question de la rupture et de la continuité entre l'histoire scolaire et l'histoire universitaire (Lautier \& Allieu-Mary, 2008).

Il montre que le travail en classe d'histoire ne saurait se réduire à un ensemble de techniques rencontrées dans les situations habituelles de classe, décrites suivant le modèle de la discipline scolaire (Chervel, 1998) qui perçoit l'histoire scolaire comme éloignée de sa discipline de référence. Certains didacticiens envisagent en effet la continuité entre les démarches de l'histoire universitaire et celles de l'histoire enseignée. Comment dès lors penser une dialectique de la continuité et de la rupture, comment envisager les conditions de l'apprentissage de la pensée historienne en classe, tout en tenant compte des contraintes de la forme scolaire (p. 51) ? À cet effet, S. Doussot propose des pistes pour penser et travailler la référence savante en classe d'histoire.

Ce travail s'intègre dans le cadre théorique de la problématisation élaboré par M. Fabre (2009). Il ne s'agirait plus d'envisager les savoirs comme le résultat de la résolution d'un problème construit par ailleurs et selon une dimension strictement propositionnelle du savoir (savoir que...). Il s'agirait plutôt d'envisager les savoirs comme étant liés à des problèmes et faisant état de nécessités (savoir qu'il ne saurait en être autrement). Ce questionnement engagerait un processus de problématisation analogue à celui des historiens quand il permet d'explorer les différents possibles qui expliquent une situation historique. Les élèves produiraient alors des hypothèses non seulement pour comprendre comment les choses se sont passées mais surtout pourquoi elles se sont passées ainsi et pas autrement (Koselleck, 1997, 